\title{
Diagnóstico de SARS CoV-2 posterior a manejo quirúrgico de acalasia: a propósito de un caso
}

\section{Diagnosis of SARS CoV-2 after surgical management of achalasia: On the subject of a case}

\author{
Elías Ballesteros-Suárez ${ }^{1 *}$, Erwin I. Marín-Pardo ${ }^{1}$, Mónica Heredia-Montaño ${ }^{1}$ y Cheryl Z. Díaz-Barrientos ${ }^{2}$
}

${ }^{1}$ Servicio de Cirugía General; ${ }^{2}$ Servicio de Coloproctología. Hospital Universitario de Puebla, Puebla, México

\begin{abstract}
Resumen
Introducción: Ante la pandemia de enfermedad por coronavirus 2019 (COVID-19) se ha puesto a prueba a los sistemas de salud y en el contexto perioperatorio surgen múltiples cuestiones que modifican la práctica habitual o requieren una atención especial. Los pacientes postoperados son otro grupo de pacientes en los que la infección por coronavirus 2 del síndrome respiratorio agudo grave (SARS-CoV-2) es un desafío diagnóstico y tiene una alta tasa de mortalidad. Caso clínico: Mujer de 61 años que ingresó al servicio de urgencias, donde se le realizó triaje respiratorio sin encontrar factores de riesgo, con intolerancia a la vía oral, disfagia a sólidos y líquidos, astenia, adinamia, pirosis y dolor retroesternal de tres meses de evolución. La panendoscopia reportó trastorno motor tipo acalasia. Fue sometida a intervención quirúrgica, posterior a la cual presentó sintomatología respiratoria. Conclusión: Es importante implementar protocolos para el manejo de pacientes con diagnóstico de COVID-19 que requieren ser sometidos a intervención quirúrgica.
\end{abstract}

Palabras clave: Acalasia. Miotomía de Heller. SARS-CoV-2. COVID-19.

\begin{abstract}
Introduction: Faced with the coronavirus disease 2019 (COVID-19) pandemic, health systems have been put to the test and in the perioperative context, multiple issues arise that modify routine practice or require special attention. Postoperative patients are another group of patients in whom severe acute respiratory syndrome coronavirus 2 (SARS-CoV-2) infection is a diagnostic challenge and has a high mortality rate. Clinical case: 61-year-old woman who was admitted to the emergency department, where respiratory triage was performed without finding risk factors, with intolerance to the oral route, dysphagia to solids and liquids, asthenia, adynamia, heartburn and retrosternal pain of 3 months of evolution. Panendoscopy reports Achalasia-type motor disorder, she underwent surgical intervention, after which she presented respiratory symptoms. Conclusion: It is important to implement protocols for the management of patients diagnosed with COVID-19 that require surgical intervention.
\end{abstract}

Key words: Achalasia. Heller myotomy. SARS-CoV-2. COVID-19.

Correspondencia:

*Elías Ballesteros-Suárez

E-mail: ebs02@outlook.com
Fecha de recepción: 28-10-2020

Fecha de aceptación: 24-11-2020

DOI: 10.24875/RHJM.20000118
Disponible en internet: 09-04-2021 Rev Hosp Jua Mex. 2021;88(1):27-31

www.revistahospitaljuarez.com 1405-9622/@ 2020 Sociedad Médico-Quirúrgica del Hospital Juárez de México, A.C. Publicado por Permanyer. Este es un artículo open access bajo la licencia CC BY-NC-ND (http://creativecommons.org/licenses/by-nc-nd/4.0/). 


\section{Introducción}

Ante la pandemia de enfermedad por coronavirus 2019 (COVID-19) se ha puesto a prueba a los sistemas de salud y en el contexto perioperatorio surgen múltiples cuestiones que modifican la práctica habitual o requieren una atención especial ${ }^{1}$. Es imprescindible entender que el término cirugía "electiva» no significa cirugía opcional y más bien implica que un procedimiento no se indica inmediatamente en respuesta a una emergencia potencialmente mortal, o que compromete la función de una extremidad. Una estimación actual sugiere que más del $50 \%$ de los casos de todas las cirugías electivas tienen el potencial de ocasionar un daño significativo en los pacientes si son canceladas o retrasadas ${ }^{2}$. Tal es el caso de la acalasia esofágica, una enfermedad poco frecuente, cuyo término proviene de 1937 y fue propuesto por Lendrum ${ }^{3}$. Es un trastorno motor esofágico primario infrecuente que tiene una incidencia de un caso por cada 100,000 habitantes en el mundo occidental. Afecta a ambos sexos por igual a cualquier edad y presenta una prevalencia mayor entre los 20 y los 50 años ${ }^{4}$. Se caracteriza por cambios en la manometría esofágica, caracterizados por la ausencia de peristaltismo esofágico y alteración de la relajación del esfínter esofágico inferior (EEI) en respuesta a la deglución ${ }^{5}$.

\section{Caso clínico}

Paciente de género femenino de 61 años de edad con los siguientes antecedentes de importancia: hipotiroidismo diagnosticado en 2015 sin tratamiento actual, colecistectomía laparoscópica en 2009 y hospitalización en 2009 secundaria a infección por virus de influenza. Actualmente jubilada, niega tabaquismo, alérgica a penicilina y cloropiramina, presentando reacción de hipersensibilidad tipo 1. Acude al servicio de urgencias, donde se le realiza triaje respiratorio sin encontrar factores de riesgo; se toma radiografía de tórax, la cual no presenta patrón COVID (Fig. 1). La paciente refiere que hace tres meses inicia con disfagia intermitente a alimentos sólidos, acompañada por regurgitación de los alimentos aproximadamente 10 minutos después de su ingesta, y dolor torácico tipo opresivo de intensidad leve (4/10), con progresión de la sintomatología en los últimos meses. Hace 12 días previos a su ingreso inicia con intolerancia a la vía oral, astenia, adinamia, pirosis y dolor retroesternal, refiere pérdida ponderal de 5 kilos en 6 semanas aproximadamente, siendo ingresada al servicio de urgencias.

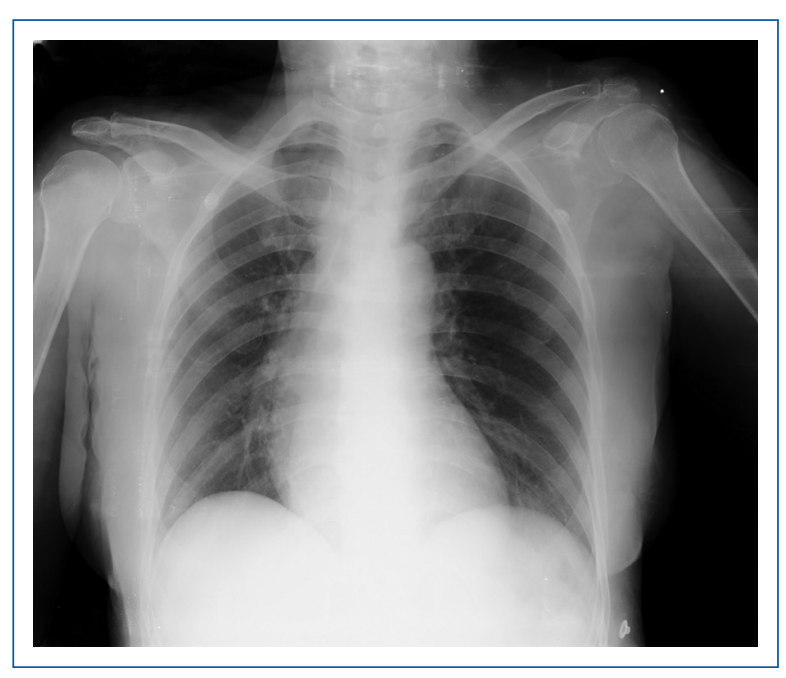

Figura 1. Radiografía de tórax sin evidencia de patrón COVID.

Exploración física con mucosa oral deshidratada, resto sin alteraciones. Debido a la sintomatología de la paciente se realiza panendoscopia, que reporta lo siguiente: tercio distal con erosiones lineales mayores de $5 \mathrm{~mm}$ confluentes a la unión, estenosis puntiforme que se vence a la presión continua de endoscopio, integrando el diagnóstico de trastorno motor tipo acalasia, esofagitis C de los Ángeles, gastropatía erosiva. Se solicita manometría esofágica de alta resolución, la cual muestra presión de relajación integrada elevada con ondas de contracción simultáneas o fallidas en el $100 \%$ de las degluciones y panpresurización en menos del $20 \%$ de las degluciones, diagnosticándose acalasia tipo I.

Por lo anterior, se realiza manejo quirúrgico mediante cardiomiotomía laparoscópica de Heller + funduplicatura tipo Dor laparoscópica + endoscopia transoperatoria (Fig. 2). En su primer día posquirúrgico presenta elevación de temperatura a $37.7^{\circ} \mathrm{C}$, tos con expectoración, y desaturación de oxígeno al aire ambiente hasta el $83 \%$, así como campos pulmonares con hipoventilación en base derecha, por lo que se realiza telerradiografía de tórax, mostrando patrón en vidrio despulido en ambas bases y lóbulos medios, con posterior tomografía computarizada (TC) de tórax que confirma patrón COVID de tipo moderado (Fig. 3) y prueba de reacción en cadena de la polimerasa positiva para el coronavirus 2 del síndrome respiratorio agudo grave (SARS-CoV-2). Es trasladada a área COVID, donde se inicia manejo, evolucionando favorablemente, tolerando la dieta blanda y sin requerir de oxígeno suplementario, por lo que es dada de alta. En el seguimiento 


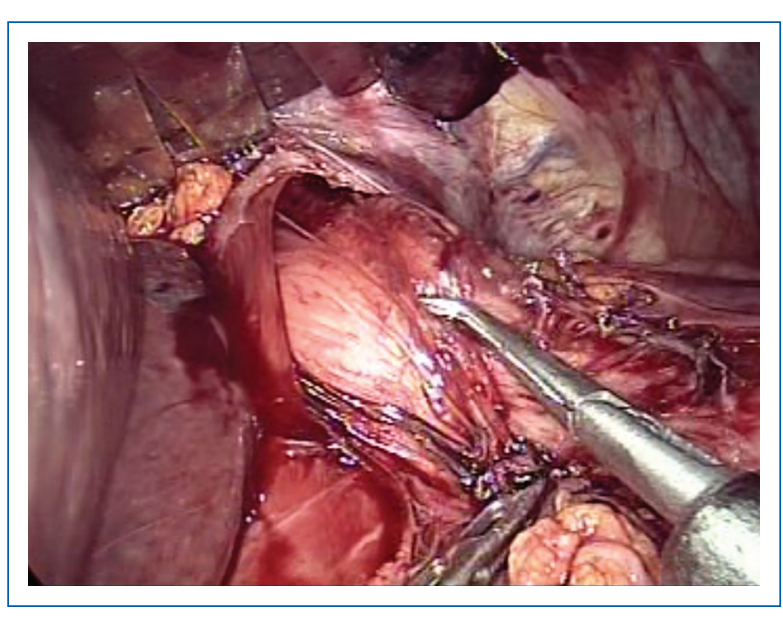

Figura 2. Miotomía laparoscópica de Heller.

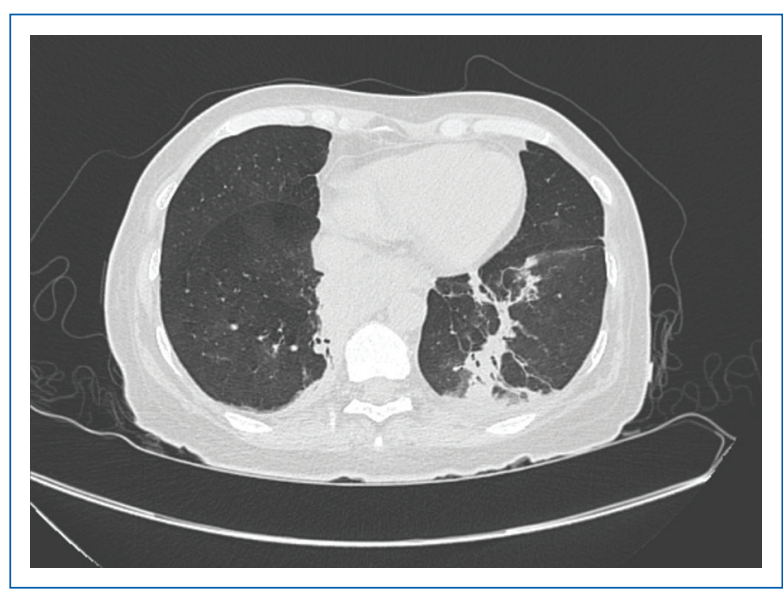

Figura 3. Tomografía axial pulmonar con múltiples opacidades en vidrio despulido de distribución difusa.

a dos semanas, con tolerancia a la vía oral y ganancia ponderal.

\section{Discusión}

La acalasia esofágica es un trastorno motor primario causado por la pérdida selectiva de las motoneuronas del plexo mientérico esofágico que ocasiona aumento en la presión basal, relajación incompleta del EEl y la desaparición de la peristalsis esofágica ${ }^{6}$. Los síntomas principales son disfagia $(90 \%)$, pirosis $(75 \%)$, regurgitación o vómito $(45 \%)$, dolor torácico no cardiaco $(20 \%)$ y pérdida involuntaria de peso $(10 \%)^{7}$. El diagnóstico se realiza sobre la base del cuadro clínico y los antecedentes, así como sobre los resultados de estudios imagenológicos y endoscópicos, dentro de los que encontramos la fluoroscopia y estudio contrastado de esófago-estomago-duodeno, la ultrasonografía endoscopia, la TC y la endoscopia superior ${ }^{8}$. La endoscopia de vías digestivas altas permite descartar enfermedad erosiva por reflujo gastroesofágico, anomalías estructurales y cáncer de esófago ${ }^{9}$. La herramienta diagnóstica de elección es la manometría esofágica de alta resolución, la cual ha demostrado superioridad diagnóstica en comparación con la manometría convencional. De acuerdo con la Clasificación de Chicago, la acalasia primaria se ha dividido en tres subtipos diferentes. En los tres tipos la presión de relajación integrada es $>15 \mathrm{mmHg}^{10}$ :

- En la acalasia tipo I, anteriormente conocida como «acalasia clásica», no se observa presurización esofágica.

- La acalasia tipo II se identifica cuando hay panpresurización esofágica (> $30 \mathrm{mmHg}$ ) en el $20 \%$ de las degluciones.

- La acalasia tipo III, también llamada «acalasia espástica», se identifica en pacientes que presentan contracciones simultáneas o prematuras en el $20 \%$ o más de las degluciones, determinado por la medición de la «integral contráctil distal» $(\mathrm{ICD})>450$ $\mathrm{mmHg} \times$ segundo $\mathrm{x} \mathrm{cm}^{10}$.

La miotomía de Heller laparoscópica se considera en la actualidad el tratamiento de elección para el alivio de la disfagia en los pacientes con diagnóstico de acalasia esofágica. Se ha demostrado que gracias a ella se logra una baja incidencia de reflujo gastroesofágico y una mejoría evidente de la calidad de vida ${ }^{11}$.

Según la evidencia actual, aunque se cree que la mortalidad por COVID-19 está entre el 1 y el $3 \%$, la mayoría de las muertes han ocurrido en pacientes de edad avanzada con afecciones cardiopulmonares subyacentes, la mayoría de ellos hipertensos, diabéticos y con obesidad. Los pacientes postoperados son otro grupo de pacientes en los que la COVID-19 es un desafío diagnóstico y tiene una alta tasa de mortalidad. Un curso postoperatorio complicado puede observarse especialmente en pacientes de edad avanzada con afecciones de salud subyacentes ${ }^{1}$.

La presentación clínica es muy variable y se han observado diversos cuadros, desde leves (con síntomas catarrales) hasta muy graves, con desarrollo de síndrome de distrés respiratorio del adulto. Existen pacientes asintomáticos, que suponen un problema a nivel epidemiológico por su capacidad de transmisión de forma desapercibida. El periodo de incubación medio es de unos 5 días (con un rango entre 0 y 
14 días), un $97.5 \%$ de los pacientes desarrollan la enfermedad en los primeros 12.5 días de incubación. En las fases iniciales de la enfermedad pueden no apreciarse alteraciones en la radiología simple, e incluso en la TC si se realiza en los primeros dos días de síntomas. Sin embargo, a medida que evoluciona el cuadro aumenta la sensibilidad de la TC, sobre todo a partir del 6. día, en el que casi todos los pacientes con COVID-19 presentan alguna alteración. Cuando la imagen radiológica es anormal existen algunos patrones comunes. En la radiografía simple de tórax se identifican zonas asimétricas de opacidad alveolar o intersticial, parcheadas 0 difusas. El patrón más habitual es el de neumonía bilateral, con opacidades en vidrio deslustrado subpleurales, con márgenes poco definidos y una ligera predilección por el lóbulo inferior derecho. Los hallazgos más característicos en la TC torácica son opacidades múltiples en vidrio deslustrado con consolidaciones en la periferia del pulmón. Si se identifican estos hallazgos atípicos (incluso en pacientes asintomáticos) se debe considerar el diagnóstico de COVID-19 ${ }^{12}$.

En el estudio de mortalidad y complicaciones pulmonares en pacientes sometidos a cirugía con infección perioperatoria por SARS-CoV-2, un estudio de cohorte internacional, la infección por SARS-CoV-2 fue diagnosticada preoperatoriamente en 294 (26.1\%) de 1,128 pacientes y postoperatoriamente en 806 (71.5\%), faltando el momento del diagnóstico en 18 pacientes. La cirugía de emergencia se realizó en $835(74.0 \%)$ de 1,128 y la cirugía electiva en 280 (24.8\%). La mortalidad a los 30 días fue del $23.8 \%$ (268 de 1,128). Los hombres tenían una mortalidad a 30 días más alta que las mujeres (28.4 vs. $18.2 \%)$. La mortalidad fue mayor después de cirugía de emergencia (25.6\%) que después de cirugía electiva (18.9\%). Las complicaciones pulmonares se asociaron con altas tasas de mortalidad a 10 días en pacientes electivos con diagnóstico postoperatorio de SARS-CoV-2 (39 de 138), pacientes de emergencia con diagnóstico preoperatorio (53 de 134) y pacientes de emergencia con diagnóstico postoperatorio de SARS-CoV-2 (125 de 290) ${ }^{13}$.

Tal como muestran los porcentajes del estudio antes mencionado, el diagnóstico de COVID-2019 es más frecuente en el posoperatorio, así mismo, las complicaciones que los pacientes pueden presentar tras una intervención quirúrgica; lo que demuestra la importancia de la detección preoperatoria del SARS-CoV-2, con la finalidad de minimizar la transmisión intrahospitalaria $y$, a la vez, reducir el riesgo de complicaciones pulmonares posoperatorias en aquellos pacientes infectados.

\section{Conclusiones}

Debido a la pandemia por la que se está atravesando no solo a nivel nacional sino mundial, el sector salud se ha tenido que enfrentar a grandes cambios, en los que destacan los servicios quirúrgicos, los cuales a raíz de la propagación de la COVID-19 se vieron en la necesidad de cancelar y/o posponer la mayoría de cirugías electivas, y en el caso de aquellos pacientes que requieren cirugía de urgencia, llevarlas a cabo con la mayor protección posible, en especial aquellos pacientes que cuentan con diagnóstico confirmatorio de infección por SARS-CoV-2. Es de suma importancia la adaptación, creación y aplicación de protocolos enfocados a obtener un diagnóstico temprano de COVID-19, sobre todo en aquellos pacientes que son sometidos a manejo quirúrgico urgente o que por su patología deben ser intervenidos lo antes posible, ya que sin los protocolos y medidas correctas se expone a todo el personal $y$ al resto de pacientes a contraer el virus.

\section{Agradecimientos}

Al personal del Servicio de Cirugía General del Hospital Universitario de Puebla.

\section{Financiamiento}

La presente investigación no ha recibido ninguna beca específica de agencias de los sectores públicos, comercial o sin ánimo de lucro.

\section{Conflicto de intereses}

Los autores declaran no tener conflicto de intereses alguno.

\section{Responsabilidades éticas}

Protección de personas y animales. Los autores declaran que para esta investigación no se han realizado experimentos en seres humanos ni en animales.

Confidencialidad de los datos. Los autores declaran que han seguido los protocolos de su centro de trabajo sobre la publicación de datos de pacientes.

Derecho a la privacidad y consentimiento informado. Los autores han obtenido el consentimiento informado de los pacientes y/o sujetos referidos en el artículo. Este documento obra en poder del autor de correspondencia. 


\section{Bibliografía}

1. Garduño López AL, Guido Guerra RE, Guizar Rangel MT, Acosta Nava VM, Domínguez-Cherit G, Álvarez Bobadilla G. Manejo perioperatorio de paciente con COVID-19. Rev Mex Anestesiol. 2020; 43(2):109-20

2. Cuencia Pardo J, Vélez Benítez E, Morales Olivera M, Iribarren Moreno R, Contreras Bulnes L, Bucio Duarte J. Reactivación de la consulta y cirugía electiva durante la pandemia: recomendaciones de seguridad. Medicina Basada en Evidencia. Cir Plast. 2020;30(1):6-21.

3. Zaninotto G, Constantini M, Rizzetto C, Ancona E. Acalasia: estrategias terapéuticas. Cir Esp. 2004;75(3):117-22.

4. Marinello FG, Targarona EM, Balangué C, Monés J, Trías M. Tratamiento quirúrgico de la acalasia: ¿mejor que las dilataciones? Gastroenterol Hepatol. 2009;32(9):653-61.

5. Allaix ME, Patti MG. Nuevas tendencias y conceptos en el diagnóstico y tratamiento de la acalasia. Cir Esp. 2013;91(6):352-57.

6. Roque González R, Martínez Alfonso MÁ, Torres Peña R, Anido Escobar V, Naranjo Hernández D, Díaz Drake Z. Miotomía de Heller laparoscópica para el tratamiento de la acalasia esofágica en el adulto mayor. Rev Cub Cir. 2015;31(4):309-17.
7. Hernández Mondragón OV, Solórzano Pineda OM, González Martínez MA, Blancas Valencia JM, Caballero Luengas C. Miotomía endoscópica peroral para el tratamiento de acalasia y otros trastornos motores del esófago. Resultados a corto y mediano plazo en un centro de referencia en México. Rev Gastroenterol Mex. 2019;84(1):1-10.

8. Jiménez Ramos R, Roque González R, Anido Escobar V. Estrategias terapéuticas en el tratamiento de la acalasia esofágica. Rev Cub Cir. 2015;31(4):318-26.

9. Germán Prieto R, Edison Prieto J, Casas F, Ballén H. Acalasia, una visión actual. Rev Colomb Cir. 2019;34(1):171-8.

10. Morales Maza J, Clemente Gutiérrez U, Santes O, Mier Terá ES, Sánchez Morales GE, Rodríguez Quintero JH, et al. Acalasia desde la perspectiva del cirujano. Rev Mex Cirug Apar Diges. 2019;8(1):94-100.

11. Roque González R, Martínez Alfonso MÁ, Jiménez Ramos $R$, Anido Escobar V, Morera Pérez M. Calidad de vida y evolución clínica después de miotomía de Heller con fundoplicatura de Dor. Rev Cub Cir. 2019;58(1):55-63.

12. Rubio Pérez I, Badia JM, Mora Rillo M, Martín Quirós A, García Rodríguez J, Balibrea JM. COVID-19: conceptos clave para el cirujano. Cir Esp. 2020;98(6):310-9.

13. COVIDSurg Collaborative. Mortality and pulmonary complications in patients undergoing surgery with perioperative SARS-CoV-2 infection: an international cohort study. Lancet. 2020;396(1):27-38. 